\title{
ROLE OF CAMERAL MUCOUS GEL IN PRIMARY NARROW ANGLE AND CLOSED ANGLE GLAUCOMA: A PATHOGENIC CLUE
}

\author{
F. JAVIER CARRERAS and FERNANDO GONZÁLEZ-CABALLERO \\ Granada, Spain
}

\begin{abstract}
SUMMARY
The cameral mucous gel (CMG) has been described as a layer of glycoprotein-enriched hyaluronic acid lining the anterior surface of the iris, covering the trabecular meshwork, and spreading over the posterior surface of the cornea. The CMG is thought to exert a colloidosmotic effect on the hydrostatic forces involved in the circulation of the aqueous humour which may help our understanding of the pathophysiology of open angle and angle closure glaucomas. The CMG was precipitated in two normal human eyes, one with an artificially shortened anterior chamber and the other with an open chamber. In the eye with a narrow angle, the CMG was seen to fill the iridocorneal gap completely, blocking access to the trabecular meshwork from the central anterior chamber. The CMG may be implicated in the pathogeny of narrow angle and closed angle glaucoma. The two types of glaucoma may share a common mechanism depending on the thickness of the layer of CMG that precedes the exit pathways. Pretrabecular CMG thickness is a decisive determinant of the colloidosmotic resistance of the gel to aqueous outflow, and this thickness is governed by, among other factors, the position of the iris relative to the posterior surface of the cornea. The formation of a thick layer of CMG in the narrow chamber angle prevents the normal anterior chamber pressure from exerting a backward displacement effect on the peripheral iris. Unopposed posterior chamber pressure may therefore force the peripheral iris forward, making angle closure likely.
\end{abstract}

In primary closed angle glaucoma, contact between the peripheral iris and the inner surface of the trabecular meshwork prevents the outflow of aqueous in the region of attachment. Since the initial work by Curran ${ }^{1}$ and Barkan, ${ }^{2}$ two mechan-

From: ${ }^{1}$ Department of Surgery (Ophthalmology), Faculty of Medicine, and ${ }^{2}$ Department of Applied Physics, University of Granada, Spain.

Correspondence to: Dr F. J. Carreras, Plaza de San Pedro 3, 41, E-04001 Almeria, Spain. Fax: +34 50236523. isms have been advocated as producing a primary closed angle glaucoma: parasympathetic inhibition and pupil block. In parasympathetic inhibition, passive pupillary dilation causes the bunched-up iris to ride against the trabecular meshwork. In pupil block, as a result of increased autonomic activity, with a shallow anterior chamber and a narrow angle, the apposition of the iris to the peripheral cornea increases the pressure of the posterior chamber relative to the anterior chamber, initiating an acute glaucoma. ${ }^{3}$

Angle closure glaucoma may occur either as a single acute attack or as several intermittent subacute attacks (also known as chronic or 'creeping' angle closure glaucoma. ${ }^{4}$ Under the influence of various physiological factors, a portion of the angle closes and tension rises to a variable degree. The angle can then open spontaneously and tension may return to normal or progress to complete closure. .6 $^{4-6}$ It has been shown that asymptomatic partial angle closure is another manifestation of angle closure in eyes at risk, but in some instances partial angleclosing mechanisms may consequently cause permanently increased intraocular pressure (IOP). ${ }^{2,7,8}$

The presence of mucosubstances rich in glycosaminoglycans (GAGs) in the aqueous pathways has been extensively debated since the work by Bàràny and Scotchbrook. ${ }^{9}$ They provided physiological evidence of the role of hyaluronidase-sensitive material in aqueous outflow resistance in bovine eyes. Those findings were soon morphologically supported by Zimmerman's ${ }^{10}$ description of a gel of mucosubstances filling the intertrabecular spaces in a human eye, at the light microscope level. Numerous studies focused on the GAGs of the trabecular meshwork, and Zimmerman's findings were disputed. $^{11}$ In spite of the difficulty of histological preservation and quantitative evaluation of mucosubstances in open cavities, much of the discussion on outflow resistance is based on the histological

Eye (1995) 9, 619-623 ㅇ 1995 Royal College of Ophthalmologists 
evidence provided by conventional methods of fixation and embedding, non-specific for mucosubstances.

Rohen and Witmer's ${ }^{12}$ description of extracellular material in the trabecular meshwork, fixed with conventional methods, centred attention on the fibrillar matrix components of the juxtacanalicular tissue. Increased synthetic activity of the trabecular meshwork cells with age and in glaucomatous eyes has been related to an increase in extracellular material in the trabecular meshwork with a concomitant thickening of the trabecular beams and accumulation of plaque material in the juxtacanalicular tissue. ${ }^{13}$ The juxtacanalicular tissue is still considered by some workers the most likely site of outflow resistance in normal eyes and eyes with open angle glaucoma. ${ }^{13,14}$

Although, at the electron microscope level, the evidence of intertrabecular GAGs has been limited to a thin layer on the surface of the trabecular cells, ${ }^{15,16}$ the osmotic role of mucosubstances in aqueous outflow resistance has received support from the use of biotinylated hyaluronan binding protein in rabbit and bovine eyes, ${ }^{17,18}$ and changes in GAGs and proteins of the aqueous humour and exit pathways have been related to the pathogeny of open angle glaucoma. ${ }^{17,19-22}$

Recently, the description of the aqueous drainage pathways in human eyes has been completed with the report of a cameral mucous gel (CMG). ${ }^{23}$ The $\mathrm{CMG}$ is a layer of proteoglycan-enriched hyaluronic acid lining the anterior surface of the iris, filling the angle recess, covering the trabecular meshwork, and spreading over the posterior surface of the cornea. The CMG has been partially characterised by lectins, and the presence in the gel of hyaluronate and chondroitin sulphate has been demonstrated by monoclonal antibodies. ${ }^{23-25}$ CMG requires special methods for mucosubstances to be elicited, and so is not to be confused with the currently described extracellular materials of the exit pathways.

The CMG is thought to exert a colloid-osmotic effect on the hydrostatic forces involved in the circulation of the aqueous humour. A theoretical model of a gel of hyaluronate filling the intertrabecular spaces has been proposed by Ethier et al. ${ }^{26}$ However, due to the pretrabecular position of CMG a remodelling of the physiology of aqueous circulation and outflow resistance may be required for the understanding of the pathophysiology of open angle and angle closure glaucomas.

In this study the osmotic effect exerted by the CMG is introduced in the current model for angle closure, ${ }^{3,27-31}$ and the mechanisms involved in the rise in IOP and closure of the angle are discussed. With this aim, CMG was precipitated in a fresh normal human eye with an artificially shortened anterior chamber. Another normal eye, fixed with the same procedure, but maintaining an open chamber, served as a reference.

\section{MATERIALS AND METHODS}

Two eyes from a healthy young male who died in a traffic accident were examined, having been fixed immediately after death. The fixative used contained $2 \%$ glutaraldehyde, $1 \%$ formaldehyde, $0.5 \%$ cetylpiridinium chloride and $0.1 \%$ copper sulphate in $0.05 \mathrm{~mol} / \mathrm{l}$ phosphate-buffered saline (PBS) at $\mathrm{pH}$ 7.4. Both eyes were fixed by perfusion of the canulated anterior chamber at a pressure of $5 \mathrm{~cm}$ $\mathrm{H}_{2} \mathrm{O}$ for 1 hour at room temperature. During fixing, one eye was maintained with the cornea upwards to keep the anterior chamber open. The second eye was positioned with the cornea downwards, so as to reduce the amplitude of the anterior chamber by the gravitational displacement of the iris-lens diaphragm, mimicking a narrow angle. After fixing, the eyes were sectioned equatorially and the anterior polar portion stained en bloc in a solution of cationised ferritin (Sigma Laboratories) containing $250 \mu \mathrm{g} / \mathrm{ml}$ in PBS at $\mathrm{pH} 7.4$ at $4{ }^{\circ} \mathrm{C}$, for 1 hour. The anterior segment was then divided into wedges containing cornea, sclera, ciliary body and iris. The pieces were embedded in plastic JB4 (Taab Laboratories), without previous dehydration. The plastic was replaced several times, to allow a complete dehydration of the tissue, over a period of 24 hours and without stirring, to avoid dispersion of CMG. After polymerisation, sections $2{ }^{\circ} \mathrm{m}$ thick were cut, revealed with potassium ferrocyanide and contrasted with nuclear red. Sections were studied and photographed at the light microscope level.

\section{RESULTS}

CMG was precipitated in the anterior chamber of both eyes at the time of fixation, and maintained a near physiological position during embedding. In the eye fixed with the cornea upwards, the anterior chamber was wide open and distribution of the gel was uniform (Fig. 1). The layer was thicker at the surface of the iris and chamber angle, and thinner at the posterior surface of the cornea. This is presumably the physiological position of the gel as an expression of the polarisation of the glycoprotein concentration in suspension in the aqueous.

The eye fixed with the cornea downwards showed a very shallow anterior chamber. This disposition mimics the narrowing of the chamber caused by the forward displacement of the lens, and the iris is almost parallel to the corneal curvature. In some quadrants the peripheral iris touched the trabecular meshwork, as shown in Fig. 2. In this eye, the distribution of the CMG was slightly different (probably as a consequence of manipulation) 


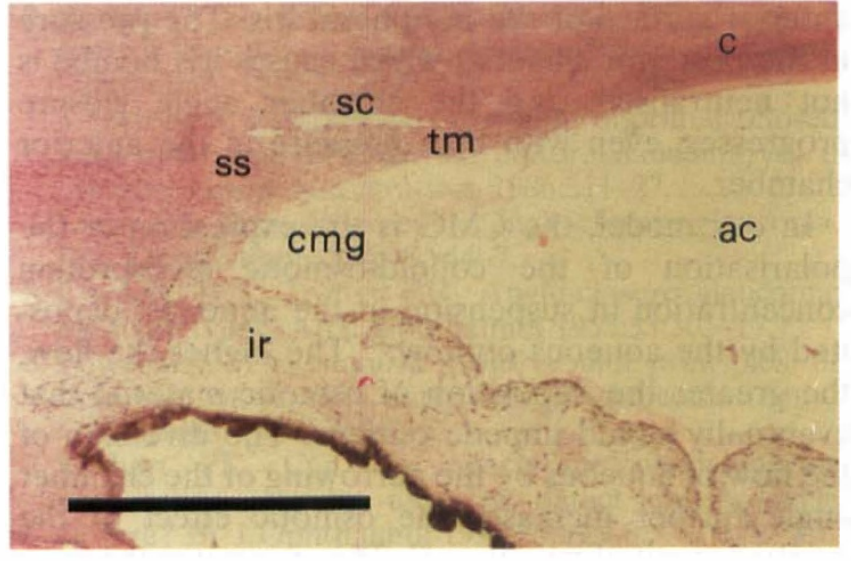

Fig. 1. Anterior chamber structures of a human eye fixed with the cornea upwards. The fixative, containing cetylpiridinium chloride, perfused the anterior chamber at a pressure of $5 \mathrm{~cm} \mathrm{H}_{2} \mathrm{O}$. The $\mathrm{CMG}$ is well preserved and maintains its physiological position, covering the anterior surface of the iris, chamber angle and corneal endothelium. Inclusion in plastic JB4; immersion in cationised ferritin; nuclear red staining. ac, anterior chamber; c, cornea; $\mathrm{cmg}$, cameral mucous gel; ir, iris root; sc, Schlemm's canal; ss, scleral spur; tm, trabecular meshwork. Scale bar represents $1 \mathrm{~mm}$.

compared with the eye with the cornea upwards. CMG was absent in some areas and had accumulated in others (Fig. 2). In general, the gel was also well preserved and close to its physiological position, although it showed a tendency to detach from the anterior iris surface. In areas where the iris was closer to the posterior corneal surface, the CMG was seen to fill the iridocorneal gap completely, blocking access to the trabecular meshwork from the central anterior chamber (Fig. 2).

\section{DISCUSSION}

Although the pathogenesis of angle closure is multifactorial and still under discussion, ${ }^{7,8}$ a few simple concepts are recognised as having a role: closure of the angle depends on the equilibrium between the pressure in the anterior chamber, which tends to push the iris-lens diaphragm backwards, and the pressure in the posterior chamber, which tends to push it forwards. This play of forces may be unbalanced by pupil dilation and pupil block, causing iris bombé. ${ }^{3}$ The lens may also be displaced anteriorly as a result of cilary muscle activity leading to a narrowing of the anterior chamber angle. . $^{32,33}$ Additionally, some structural characteristics have been detected in eyes with angle closure glaucoma. Irrespective of race, eyes with primary angle closure are shorter and have a shallower anterior chamber than age-matched normal eyes. Shallowing of the anterior chamber has been related to a relatively forward position of the lens, a normal or more than average lens thickness for age, and progressive lens thickening as a result of continued lens growth with age. ${ }^{34,35}$ The closure of the chamber angle is thought

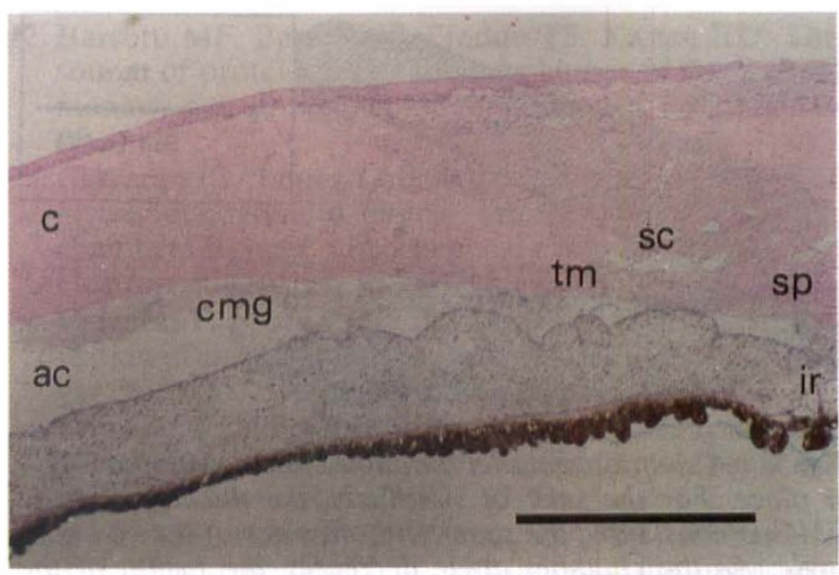

Fig. 2. Anterior chamber structures of a human eye fixed with the cornea downwards. Same procedure as in Fig. 1. Perfusion pressure is not enough to oppose the displacement of the iris-lens diaphragm, and the anterior chamber appears very narrow. The CMG is partially displaced from its physiological position, and has been removed from some areas. In areas where the iris approaches the cornea, the CMG completely fills the gap. In this situation, access to the trabecular meshwork from the central anterior chamber is blocked by the gel, although the iris does not touch the corneal endothelium. Inclusion in plastic JB4; immersion in cationised ferritin; nuclear red staining. ac, anterior chamber; c, cornea; $\mathrm{cmg}$, cameral mucous gel; ir, iris root; sc, Schlemm's canal; sp, scleral spur; tm, trabecular meshwork. Scale bar represents $1 \mathrm{~mm}$.

to start in the superior sector and to progress both nasally and temporally, completing closure at the 6 o'clock position..$^{31}$ Primary angle closure probably starts in the angle recess and extends anteriorly and circumferentially, as deduced from the distribution of peripheral anterior synechiae with pressure gonioscopy. ${ }^{31}$

To the mechanisms described above can be added a possible role of mucosubstances in the aqueous outflow. Some of the ubiquitous GAGs and proteoglycans of the extracellular matrix of the connective tissue are present in the $\mathrm{CMG}^{23-25}$ Proteoglycans are known to play a major role in the flow of solvents in the extracellular matrix through their colloidosmotic effect. ${ }^{36}$ From perfusion experiments and fluorophotometry studies in bovine, rabbit and monkey eyes, it appears that plasma-derived proteins may also play a role in the resistance of aqueous outflow. These proteins are reported to enter the anterior chamber through the iris root. ${ }^{20-22}$ It is likely that proteins in the aqueous humour generate a fraction of outflow resistance in combination with the exit pathways' GAGs. ${ }^{17}$ The lining of the anterior chamber by the CMG is especially thick at the chamber angle and, because of its pretrabecular position, it can contribute to some extent to the triggering mechanism of ocular hypertension, by means of its colloid-osmotic effect in aqueous outflow resistance. ${ }^{23}$

Our results show that, when the iris approaches the posterior surface of the cornea, the mucous layer 




Fig. 3. A simplified model for angle closure with the CMG in place. For the sake of simplicity, the thickness of the $C M G$ is considered the same on both sides of the chamber angle. As the chamber angle decreases, the fusion of the $C M G$ layers increases the extent of the chamber angle occupied by the gel. $\mathrm{d}$, width of the CMG at the recessus angularis; th, thickness of the CMG on the chamber walls; $\alpha$, chamber angle.

on the anterior surface of the iris tends to merge with the layer on the endothelial surface. The eventual fusion of the two layers markedly increases the thickness of the pretrabecular CMG to be percolated by the aqueous humour before leaving the anterior chamber (Fig. 2). A simple theoretical model of the closure of the angle is illustrated in Fig. 3, where the increase in thickness of the pretrabecular CMG is related to the size of the chamber angle. The thickness of the gel in the recessus angularis $(d$ in Fig. 3) is inversely proportional to the sine of half the value of the chamber angle ( $\alpha$ in Fig. 3):

$$
d=t h / \sin (\alpha / 2)
$$

where $t h$, the thickness of the gel in the chamber walls, is assumed for the sake of simplicity to be the same on both sides.

According to Ethier, ${ }^{26}$ the flow resistance in a concentration-polarised layer of a gel of hyaluronic acid placed in the intertrabecular spaces of the trabecular meshwork arises primarily from the osmotic pressure within the gel. A relationship exists between the characteristic thickness of such a layer, $d$, the diffusion coefficient of the polarised substance, $D$, and the flow velocity through the gel, $U$ :

$$
d \sim D / U
$$

The greater the value of $d$ the smaller the velocity of flow through the gel. A value of $d$ exists that impedes completely the flow of solvent through the gel.

In this model of angle closure, as the angle becomes narrower and $d$ increases, a value of $d$ can be reached at which the flow is stopped. The aqueous is prevented from leaving the anterior chamber through the portion of the narrowed chamber angle with an increased thickness of the pretrabecular CMG. Although the pressure in the anterior chamber can rise steeply, in the area of contact of the CMG the pressure head does not reach the anterior surface of the peripheral iris. The pressure in the posterior chamber which causes iris bombé is not neutralised, and the chamber angle closure progresses, even with high pressure in the anterior chamber.

In our model, the CMG is the expression of the polarisation of the colloid-osmotic glycoprotein concentration in suspension in the aqueous, deposited by the aqueous outflow. ${ }^{23}$ The higher the flow, the greater the deposition of osmotic material that eventually would impede outflow. The diversion of the flow of aqueous by the narrowing of the chamber angle further increases the osmotic effect of the newly deposited mucous gel. This promotes the nonequilibrium of pressures in adjacent areas, and favours the narrowing and eventual closure of the remaining chamber angle. Nevertheless, a stable situation is theoretically possible in which the chamber angle is narrow, although open, and the IOP is elevated because of the thick mucous gel with no further angle closure. This would define the pathogenesis of narrow angle glaucoma. Equilibrium depends on many structural and functional factors, among which rates of production and elimination of osmotic material are of primary significance.

Finally, perfusion of enucleated eyes with serum caused a decrease in the facility of outflow attributed to adsorption of serum proteins in the aqueous outflow system $^{37}$ or elimination of the wash-out phenomenon. $^{20}$ The osmotic behaviour of the CMG largely depends on proteins in the aqueous. Since colloid-osmotic pressure inside a gel of GAGs increases sharply with the presence of proteins, ${ }^{38,39}$ a high level of proteins in the aqueous has been implicated in the pathogenesis of glaucoma secondary to uveitis. ${ }^{34}$ Increased colloid-osmotic pressure in the CMG may also be a contributory factor in inflammatory closed angle glaucoma, as reported following panretinal photocoagulation, uveitis and scleritis. ${ }^{40}$

In conclusion, the CMG may be implicated, because of its position lining the anterior chamber, in the pathogenesis of narrow angle and closed angle glaucoma. The two types of glaucoma may share a common triggering mechanism depending on the thickness of the layer of CMG that precedes the exit pathways. Pretrabecular CMG thickness is a decisive determinant of the colloid-osmotic resistance of the gel to aqueous outflow, and this thickness is governed by, among other factors, the position of the iris relative to the posterior surface of the cornea.

Key words: Angle closure mechanisms, Aqueous proteoglycans, Closed angle glaucoma, Narrow angle glaucoma.

\section{REFERENCES}

1. Curran EJ. A new operation for glaucoma involving a new principle in the aetiology and treatment of chronic primary glaucoma. Arch Ophthalmol 1920;49:131-40. 
2. Barkan O. Glaucoma: classification, causes and surgical control. Results of microgonioscopic research. Am J Ophthalmol 1938;21:1099-117.

3. Mapstone R. Pathological physiology of primary closed angle glaucoma. In: Cairns JE. editor. Glaucoma, vol. I. London: Grune and Stratton, 1986:311-27.

4. Lowe RF. Primary creeping angle-closure glaucoma. Br J Ophthalmol 1964;48:544-7.

5. Chandler PA, Trotter RR. Angle-closure glaucoma: subacute types. Arch Ophthalmol 1955;53:305-17.

6. Lowe RF. The natural history and principles of treatment of primary angle closure glaucoma. Am J Ophthalmol 1966;61:642-51.

7. Wishart PK. Can the pilocarpine phenylephrine provocative test be used to detect covert angle closure? Br J Ophthalmol 1991;75:615-8.

8. Wishart PK. Does the pilocarpine phenylephrine provocative test help in the management of acute and subacute angle closure glaucoma? $\mathrm{Br} \mathrm{J}$ Ophthalmol 1991;75:284-7.

9. Bàràny $\mathrm{EH}$, Scotchbrook $\mathrm{S}$. Influence of testicular hyaluronidase on the resistance to flow through the angle of the anterior chamber. Acta Physiol Scand 1954;30:240-8.

10. Zimmerman LE. Demonstration of hyaluronidase sensitive acid mucopolysaccharides in trabecula and iris in routine paraffin sections of adult human eyes. Am J Ophthalmol 1957;44:1-4.

11. Duke JR, Siegelman S. Acid mucopolysaccharides in the trabecular meshwork of the chamber angle. Arch Ophthalmol 1961;66:399-404.

12. Rohen JW, Witmer R. Electron microscopic studies on the trabecular meshwork in glaucoma simplex. Graefes Arch Clin Exp Ophthalmol 1972;183:251-66.

13. Lütjen-Drecoll E, Shimizu T, Rohrbach M, Rohen JW. Quantitative analysis of 'plaque material' in the inner and outer wall of Schlemm's canal in normal and glaucomatous eye. Exp Eye Res 1986;42:443-55.

14. Buller C, Johnson D. Segmental variability of the trabecular meshwork in normal and glaucomatous eyes. Invest Ophthalmol Vis Sci 1994;35:3841-5.

15. Segawa K. Localisation of acid mucopolysaccharides in the human trabecular meshwork. Jpn J Clin Ophthalmol 1970;24:363-7.

16. Grierson I, Lee WR, Abrahams S. The appearance of the outflow apparatus of the eye after staining with ruthenium red. Acta Ophthalmol (Copenh) 1977; $55: 827$.

17. Gong H, Underhill CB, Freddo TF. Hyaluronan in the bovine ocular anterior segment, with emphasis in the anterior pathways. Invest Ophthalmol Vis Sci 1994;35:4328-32.

18. Lütjen-Drecoll E, Schenholm M, Tamm E, Tengblad A. Visualization of hyaluronic acid in the anterior segment of rabbit and monkey eyes. Exp Eye Res 1990;51:55-63.

19. Tamm ER, Lütjen-Drecoll E. Advances in morphologic glaucoma research. Curr Opin Ophthalmol 1992; 3:141-8.

20. Johnson M, Gong H, Freddo TF, Ritter N, Kamm R. Serum proteins and aqueous outflow resistance in bovine eyes. Invest Ophthalmol Vis Sci 1993;34:354957.

21. Freddo TF, Bartels SP, Barsotti MF, Kamm RD. The source of proteins in the aqueous humor of the normal rabbit. Invest Ophthalmol Vis Sci 1990;31:125-37.
22. Barsotti MF, Bartels SP, Freddo TF, Kamm RD. The source of protein in the aqueous humor of the normal monkey eye. Invest Ophthalmol Vis Sci 1992;33:58195.

23. Carreras FJ, Lopez-Caballero J, Porcel D. A gel of glycosaminoglycans lining anterior and posterior chambers in man: histochemical evidence at light and electron microscopy level. Eye 1992;6:574-82.

24. Carreras FJ, Porcel D, Lopez-Caballero JJ. Characterization of proteoglycans in the human cameral mucous gel by lectins. Ophthalmic Res 1994;26:275-82.

25. Carreras FJ, Porcel D. Chondroitin sulfate is a major proteoglycan constituent of human cameral mucous gel. Ophthalmic Res 1995;27:53-6.

26. Ethier CR, Kamm RD, Palaszewsky BA, Richardson TM. Calculations of flow resistance in the juxtacanalicular meshwork. Invest Ophthalmol Vis Sci 1986;127:1741-50.

27. Bhargava SK, Leighton DA, Philips CI. Early angleclosure glaucoma: distribution of iridotrabecular contact and response to pilocarpine. Arch Ophthalmol 1973;89:369-72.

28. Mapstone R. Mechanisms in ocular hypertension. Br J Ophthalmol 1979;63:325-30.

29. Høvding G, Aasved H. Angle closure glaucoma. Curr Opin Ophthalmol 1992;3:149-53.

30. Greenidge KC. Angle-closure glaucoma: a review of mechanisms. Curr Opin Ophthalmol 1991;2:133-9.

31. Inoue $T$, Yamamoto $T$, Kitazawa $Y$. Distribution and morphology of peripheral anterior synechiae in primary angle-closure glaucoma. J Glaucoma 1993;2:1716.

32. Abramson DH, Chang S, Coleman J. Pilocarpine therapy in glaucoma: effects in anterior chamber depth and lens thickness in patients receiving longterm therapy. Arch Ophthalmol 1976;94:914-8.

33. Lütjen-Drecoll E. Kaufman PL. Morphological change in primate aqueous humour formation and drainage tissues after long-term treatment with antiglaucomatous drugs. J Glaucoma 1993;2:316-28.

34. Lowe RF. Causes of shallow anterior chamber in primary angle closure glaucoma: ultrasonic biometry of normal and angle-closure glaucoma eyes. Am J Ophthalmol 1969;67:87-93.

35. Salmon JF, Swanevelder SA, Donald MA. The dimensions of eyes with chronic angle-closure glaucoma. J Glaucoma 1994;3:237-43.

36. Jackson RL, Busch SJ, Cardin AD. Glycosaminoglycans: molecular properties, protein interactions and role in physiological processes. Physiol Rev 1991;71:481-539.

37. Epstein DL, Hashimoto JM, Grant WM. Serum obstruction of aqueous outflow in enucleated eyes. Am J Ophthalmol 1978;86:101-5.

38. Ogston AG. The biological functions of the glycosaminoglycans. In: Balazs EA, editor. Chemistry and molecular biology of the intercellular matrix, vol. 3 . New York: Academic Press, 1970:1231-40.

39. Preston BN, Davies M, Ogston AG. The composition and physicochemical properties of hyaluronic acids prepared from ox synovial fluid and from a case of mesothelioma. Biochem J 1965;96:449-71.

40. Greenidge KC. Angle closure glaucoma: a review of mechanisms. Curr Opin Ophthalmol 1991;2:133-9. 